\title{
Cultural Diversity in the Meaning of Lies, Deceptions, and other Misrepresentations
}

\author{
Daniel J. Hruschka \\ School of Human Evolution and Social Chane \\ Arizona State Unversity \\ dhruschk@asu.edu
}

You are a passenger in a car driven by a close friend, and he hits a pedestrian. You know that your friend was going at least thirty five miles per hour in a zone marked twenty. There are no witnesses. Your friend's lawyer says that if you testify under oath that your friend's speed was only twenty miles per hour then you would save your friend from any serious consequences. What would you do?

People can vary dramatically in how they think about lying and deception. Consider the above dilemma used by Fons Trompenaars and Charles Hampden-Turner (1998) to probe crosscultural communication in international corporations. When Trompenaars and Hampden-Turner posed this dilemma to white collar workers from around the world, they found dramatic differences across countries (Trompenaars, 1998). In the U.S. and Switzerland, over $90 \%$ of respondents preferred telling the truth over helping their friend. In Venezuala, by contrast, the majority $(70 \%)$ preferred helping their friend with false testimony. These country-level differences are striking, but in Greece, Russia, and China there was also substantial disagreement within countries, with responses choosing either option close to $50 \%$. 
A number of factors might account for this variation in one's willingness to lie for a friend. These include one's general commitment to helping friends, trust in the judiciary, or simply concerns about getting caught (Hruschka, 2010). Because responses were based on selfreports, they may also reflect cross-cultural differences in the tendency to respond in socially desirables ways. Notably, this would constitute cross-cultural variation in another form of lying - a respondent's tendency to misrepresent to the researcher their real preferences.

The passenger's dilemma illustrates substantial diversity in people's willingness to lie, but we can also disagree on what actually counts as a lie or deception. Consider a husband who tells his wife that he's going to visit his younger brother, when instead he plans to go out drinking alone. If his wife unknowingly spreads this misinformation to her mother, would her statement also be considered a lie? Most Americans polled would say no (Danziger, 2010). However, when Eve Danziger asked a similar question of her Mopan Maya informants from Southern Belize, they uniformly classified the wife's mistaken report as a lie (or tus). When the plot thickens and the husband accidently runs into his younger brother on the way to the bar, the Mopan Maya informants no longer consider his initial statement a tus, as the husband had unintentionally told the truth!

According to Danziger, the Mopan Maya's responses hinge on their definition of tus. Specifically, what counts in the Mopan case is whether the actor has conveyed something untrue, and not what the actor actually knew or intended at the time. This Mopan view of deception extends to stories and dramatic reenactments which are enjoyed only because people believe them to represent actual historical events. For example, when Danziger brought the "Jungle Book" film to the field, her informants greatly enjoyed it. But when she informed a Mopan friend that the film was not based on actual events, the friend immediately condemned it as tus 
(Danziger, 2010). I should add that when I described these Mopan results to a colleague in Bangladesh, she found it difficult to believe, precisely because her own usage of the term "lie" (mitha) is very close to the one used in the U.S. Thus, in the face of striking differences such as those found between Mopan and U.S. classifications of lies, people from cultures on the other side of the world — Bangladesh and the U.S. — can converge quite closely in their views.

These examples suggest that there is an exciting range of variation in what counts as deception and how it is evaluated, and they highlight how we cannot be sure that definitions developed by social scientists, linguists and philosophers apply equally well across all linguistic and cultural contexts (Henrich, Heine, \& Norenzayan, 2010). In this chapter, I review the known range of variation in how people categorize and evaluate misrepresentations as well as the key models used to study it.

\section{Ways of Defining a Lie}

Researchers have used three primary approaches — classical set models, prototype models, and context-based models - to characterize lay models of lying and deception. Classical set models define a list of necessary and sufficient conditions for categorizing something as a lie or deception. For example, one simple model would define a lie as any statement satisfying the following conditions: (1) the speaker knows the statement to be false, (2) the speaker's intent is for another person to believe the statement, and (3) the statement is indeed false. Anything satisfying all three criteria would be a lie; anything else would not. Scientists frequently set up their own lay models of lying and deception using necessary and sufficient conditions to decide what to include and exclude in their research (Vrij, 2000). Similarly, philosophers have a long tradition of developing definitions of lying and deception, aiming for definitions which are 
logically consistent and which also fit the lay models we apply in everyday situations (Carson, 2006; Mahon, 2008) (Arico \& Fallis, 2013).

Like classical set models, prototype models assume that there is a list of criteria that people use to categorize a lie or deception (Coleman \& Kay, 1981). However, membership in a category is graded, so that each criterion gives additional weight to membership in the "lie" category. Thus, people can judge any specific utterance (e.g. "telling your spouse that he or she looks good in that outfit") to be more or less like a lie, based on specific facts about that utterance. Let's consider the three criteria I just used to illustrate the classical set model, (1) the speaker believes the statement to be false, (2) the speaker's intent is for the listener to believe the statement, and (3) the statement is indeed false. Coleman and Kay (1981) argued that a prototypical lie in English contains these three basic features - [+belief], [+intent], [+false]—but that the three features may influence category membership to differently weighted degrees. When asked about specific incidents, people indeed judged acts that involve more of these three features as more like lies. The most important feature, however, was whether the sender believed the statement to be false, followed by the sender's intent to deceive. Interestingly, the least important feature among English speakers was whether the information was actually false.

Prototype models of lying are not limited to these three criteria. For example, Grice (1989) has argued that omitting important details of an event or obscuring the description of a situation violates a key maxim of cooperative communication — the clarity principle. Such violations may also shape people's judgments of whether statements are lies or not.

While prototype models add important nuance to classical set models, they also face challenges. Bluffing in poker, story-telling, joking around, performing magic tricks, and perpetuating a child's belief in the Tooth Fairy all share the features of lies outlined by Coleman 
and Kay. However, few English speakers would call them lies. To address this issue, Eve Sweetser (1987) outlined an alternative "context-based" model of a lie as a false statement made in a simplified, prototypical context . In the U.S. setting, for example, a lie requires a context where the speaker: (1) is expected to help the listener or at least do no harm, and (2) the speaker believes that that true information will actually be helpful. Thus, in situations when the truth is irrelevant (and thus not helpful), such as joking around, story-telling or magic tricks, even something satisfying all or many of Coleman and Kay's criteria might not be considered a lie. Similarly, in an adversarial setting, such as poker, sports, and even politics, certain kinds of feints and bluffs would not be considered lies or deceptions because there is no expectation that the speaker aims to help the listener.

These formal models of lying and deception have focused primarily on how people categorize lies, rather than how people evaluate them as good or bad. Most studies of evaluation have adopted, often implicitly, something like a prototype methodology where they manipulate features of statements and then examine how the specific features effect people's evaluations of lies as either good or bad (McCornack et al. 1992). From the limited prior work that simultaneously examines both categorization and evaluation of misrepresentations, it is difficult to determine how evaluations might impinge on categorizations and vice versa (Fu, Lee, Cameron, \& Xu, 2001). For this reason, I examine categorization and evaluation separately.

\section{Diversity in the categorization of misrepresentations}

The Mopan Maya case illustrates the very different ways in which people can categorize lies across cultures. English speakers attend to several factors, including the speaker's intent and belief, and to a lesser extent the actual falsity of the statement. The same is true of Spanish 
mentira in Ecuador (Hardin, 2010) and Spain (Eichelberger, 2012) and Arabic kathaba in Saudi Arabia (Cole, 1996). In the Mopan case, however, all that matters is the third criteria, and thus all that is required is a simple reweighting of Coleman and Kay's three criteria: $[+$ belief $]=0$, $[+$ intent $]=0,[+$ false $]=1$. According to Sweetser's contextual model of an English lie, a lie is an untruth in a specific context, and there are many contexts where an untruth would not be a lie. These include joking around, magic tricks and bluffing. In the Mopan case, by contrast, all contexts appear to be a context where an untruth is a lie. So despite its apparent exoticness, the Mopan case can fit a simple application of both prototype and context-based models, without a need for additional criteria.

More interesting differences arise when we travel to China, and consider how the motivation for misrepresentation can actually change how it is categorized. Most research on Chinese cultural models of lying focus on lies motivated by humility—downplaying or hiding one's good deeds to be modest. A number of studies in both Mainland China and Taiwan have shown that Chinese children, like U.S. children, consistently categorize such false statements of modesty as 'lies' (Fu et al., 2010; Lee, Xu, Fu, \& Cameron, 2001). North American adults similarly nearly always categorize modesty-motivated misrepresentations as lies (Lee \& Ross, 1997; Lee et al., 2001). However, Chinese adults show a different pattern. When shown similar scenarios, nearly half of Chinese adults do not categorize the modesty-motivated misrepresentations as lies (Fu et al., 2001). In another study, Chinese working class mothers uniformly categorized videos of modesty-motivated misrepresentations as non-lies (Wang, Bernas, \& Eberhard, 2012).

The tendency for Chinese adults to classify modesty-based misrepresentations as non-lies does not extend to all positively-valued social goals. Although misrepresentations to defuse 
tension in an interaction have been unanimously judged as non-lies among Chinese working class mothers (Wang et al., 2012), misrepresentations to make someone else feel better (e.g. false compliments), to provide excuses, or to coax a child to do something for the child's benefit are variously categorized as lies or non-lies (Wang et al., 2012). And misrepresentations aimed at helping a collective over an individual are classified as lies by both Chinese adults and children, even though they can be positively evaluated (Xu, Luo, Fu, \& Lee, 2009). Thus, not all positive social goals shape the categorization of lies in the same way.

Wang et al.'s (2011) recent observational study of child-rearing practices among 40 Chinese working class mothers sheds valuable light on the ways that parents socialize their children into this complex world of lies and non-lies. Analyzing more than 12 hours of recorded interaction for each mother-child pair, the researchers found that mothers initiated discussions about the importance of telling the truth more than all but one other moral theme - diligence. Indeed, mothers initiated discussions of honesty more than respect, caring, sharing or harmony. Mothers were also more likely to respond immediately to a child's dishonesty than to transgressions in any of these other domains, and they used a number of different exemplars and models to emphasize the importance of honesty. Ironically, mothers also violated the principle of honesty more frequently than any moral theme in front of their child. A potential reason for this discrepancy arises when we consider the kinds of misrepresentations that mothers were making, and how they felt about those misrepresentations. Over $80 \%$ of the violations were aimed at one of two goals - maintaining modesty and defusing tension. And when the mothers viewed fictional videos of these kinds of transgressions, they rarely categorized those misrepresentations as lies (Wang et al., 2012). Thus, mothers appear to enact their own cultural 
model of misrepresentations for their child, engaging regularly in misrepresentations that are not considered lies while avoiding those that are.

The way in which a misrepresentation is conveyed - whether explicitly or by omissionmay also shape how misrepresentations are categorized in different cultural contexts. Consider violations of Grice's maxim of clarity (1989) — unclear statements and omissions of important facts (Grice, 1989). Students in the U.S. were substantially more likely to classify a statement violating this maxim as "deceptive" than Korean (McCornack, Levine, Solowczuk, Torres, \& Campbell, 1992; Park \& Ahn, 2007) or Hong Kong Chinese students (Yeung, Levine, \& Nishiyama, 1999). If further corroborated, this finding would suggest that Grice's maxim of clarity is another criterion which may be important in distinguishing lies from non-lies in the U.S., but that may not matter in other settings.

To summarize, a number of criteria can influence the categorization of a misrepresentation as a lie, and they influence that assessment in varying degrees. In addition to the traditional criteria of intent, belief, and falsity (Coleman \& Kay, 1981), the specific expectations in a given context (e.g., help a listener with correct knowledge) can determine whether a misrepresentation is a lie (Sweetser, 1987). In addition to this, other criteria, such as the social motivations underlying a misrepresentation and the manner in which the misrepresentation is conveyed, can influence its status as a lie. The degree to which these criteria are used varies considerably, however, depending on cultural context. There does not appear to be a single set of criteria defining a "lie" that can be applied universally across human societies.

\section{Diversity in the evaluation of lies and deception}


Once misrepresentations are categorized as lies or deceptions, people variously evaluate them more or less positively (Heyman, Sweet, \& Lee, 2009; Lee \& Ross, 1997). Religious texts across major world religions have conceded certain kinds of lies are acceptable, including modesty-motivated lies, lies in battle, and lies to bring about peace (Bok, 1978; Cole, 1996). And although lying generally has a negative moral valence in U.S. culture, in certain situations telling a lie can be viewed more positively than telling the truth. Consider lying to the Gestapo about the location of a family of Jews hiding in your house. Someone who places a strong value on the life and well-being of all humans would likely see the lie as good (Sweetser, 1981).

Multiple factors contribute to evaluations of a lie as more or less negative. Studies with both Chinese and U.S. participants indicate that lies told to help a listener are viewed more positively than those told to help oneself, which in turn are viewed more positively than those told to harm the listener (Ma, Xu, Heyman, \& Lee, 2011; Seiter, Bruschke, \& Bai, 2002; Xu et al., 2009). Moreover, more harmful lies are considered worse than less harmful lies (Levine, Asada, \& Lindsey, 2003).

While some factors affect the evaluation of lies across a range of cultures (e.g., helpfulness to the listener), their importance can vary across populations. The same modestymotivated effects observed in the categorization of lies among Chinese adults also influence their evaluation. Modesty-motivated falsehoods may be categorized as lies by children and adults in the U.S. and China (Fu et al., 2010; Fu et al., 2001; Lee \& Ross, 1997). However, even when Chinese children and adults classify modesty-motivated misrepresentations as lies, they evaluate these lies more positively than do U.S. children and adults (Fu et al., 2001; Lee, Cameron, Xu, Fu, \& Board, 1997; Lee et al., 2001). Modesty-motivated lies become increasingly acceptable to 
Chinese children as they get older (Lee et al., 1997), and indeed by the age of 11 they are considered as acceptable as immodest truths (Fu et al., 2010; Fu et al., 2001).

A related cross-cultural difference arises for lies that help a collective but harm oneself (Fu, Xu, Cameron, Heyman, \& Lee, 2007), which are commonly considered lies by Chinese respondents, but are are evaluated positively. Blum synthesizes a wide body of work on lying and collectivism in China, suggesting that these cross-cultural differences arise based on different expectations about what is helpful to the listener (Blum, 2007). Sweetser (1987) proposes that accurate information is considered most helpful in the U.S. situation. This creates a set of expectations about "good" behavior which are very similar to Grice's guidelines for "cooperative" communication — be truthful, be as informative as required but not more, be relevant, and be clear (Grice, 1989). For Chinese adults, however, being cooperative may depend on other important principles of social interaction, such as making your partner feel good and being friendly, what George Lakoff calls a "Rule of Politeness" (Lakoff, 1973). In some Western theories of communication, such as Grice's "Cooperative Principle," one helps by making sure one's partner gets the best available information (Grice 1989). In the Chinese context, one frequently helps most by making sure one's partner feels good.

In contexts defined by Grice's Cooperative Principle, Lakoff's Rules of Politeness, and Sweetser's (1987) helping principle, lies are evaluated in terms of their helpfulness to the listener. A third kind of context expects no help for the listener. In such contexts, the listener is one's competitor, and lying, deceit, and trickery are tools in the competition (Condon \& Yousef, 1974). In such contexts, clever lying and deceit can be highly valued and even praised. In the U.S., this "Competitive Principle" arises most commonly in games, sports, and perhaps politics. 
Bluffs in poker, pump fakes in football, feints in boxing are all examples of praiseworthy deceptions (and perhaps not even considered deceptions!).

The Competitive Principle does not imply a free-for-all, however. One must still abide by the rules of the game or sport. You can't pay off the referee, you can't falsely lose to win a bet, and you shouldn't try to confuse the referee through fake dives and injuries. However, within the rules of engagement set for a specific sport or game (which may also include implicit norms of good behavior), one may (and should) use deception and lying as a tool to win. In short, the Competitive Principle states: 1) follow the rules of engagement, but 2) do what it takes to win. While the Competitive Principle is confined to particular contexts in the U.S., anthropologists have noted that it may also be a more common day-to-day communicative context in other societies where there is continued and ongoing competition among individuals (Friedl, 1962; Gilsenan, 1976; Handmann, 1983). The limited ethnographic data on the topic make it difficult to judge whether there is any society where it actually is the default principle of communication in a society.

As illustrated by the above examples, evaluation of misrepresentations depends on many of the same criteria used to categorize them. However, evaluation and categorization may differ in one key respect. The criteria for categorizing a misrepresentation as a lie usually focus on the speaker-listener relationship, while the criteria for evaluating misrepresentations already identified as lies can go well beyond that. As examples, lying to the Gestapo is good because it would help save a family's life, but it is nonetheless a lie (Sweetser, 1987). Lying to help a collective over oneself is good because it helps the collective (Fu et al., 2007). And lying, deceiving, and concealing information are frequently justified in the name of national security. 
None of these evaluations are based on the speaker-listener relationship, and none would require characterizing these incidents as something other than lying.

In the above examples, the evaluation of lies depends on the consequences of those lies. Psychological research provides a useful illustration of recent debates about whether the consequence to the listener should or can be left out of consideration when evaluating a lie. Psychology has a long history of deceiving research participants in order to understand how contexts influence behavior. More than $50 \%$ of the studies published in the Journal of Experimental Social Psychology in 2002 used some form of deception, most commonly involving false information about the study's purpose, the study stimuli, the identify of coparticipants, and participants' performance (Hertwig \& Ortmann, 2008a, 2008b). Because deception is potentially a very powerful way to quickly manipulate contexts in psychologically compelling ways, it is considered a valuable tool for discovering scientific truths about behavior.

The field of behavioral economics, on the other hand, does not tolerate deception, and many experimental economics journals will not publish experiments involving deception (Ariely $\&$ Norton, 2007). The primary argument on this side is that deceiving members of the participant pool will lead them to distrust what the researcher says in future studies, making it unclear whether the results of future studies reflect honest responses to a researcher's instructions versus responses to an expected lie (Davis \& Holt, 1993). In other words, the experimental economics stance aims to ensure that Grice's maxims — be truthful, be suitably informative, be relevant, and be clear-still hold in the experimental setting, so that participants continue: (1) to act as if what the researcher says is true and (2) to feel an obligation to accurately represent their own preferences, intentions, and beliefs to the researcher. 
Interestingly, arguments from both sides focus on what the suitable way is to find the truth about human behavior, rather than about the well-being of the listener (i.e., study participant). Rather than focusing on the listener-speaker relationship, what appears to influence evaluation of lies in this case, and the others listed above, are the specific goals and values (e.g., to preserve life, to help the collective, to ensure national security, to gain scientific truth) affected by the lie.

Open Questions and Conclusions: In the last 30 years, work by linguists, psychologists, and anthropologists has identified remarkable and tantalizing differences in the ways that people think about misrepresentations. Current work has shown that people differ in the criteria and contexts they use to categorize misrepresentations as lies. Compared to categorization, the evaluation of lies involve far more inputs, including how the lie affects people, groups, and goals outside the speaker-listener pair. I finish by posing three questions raised by these findings.

How do evaluation and categorization interact? Existing studies suggest that evaluation and categorization of lies appear to involve two different kinds of decision. For example, Xu et al. (2009) showed that the categorization of a misrepresentation as a lie depends on the goal of the communication setting. Specifically, an untrue statement is categorized more like a lie in an informational setting than in a politeness setting. But the moral evaluations of the lies did not depend on expectations of the context. Rather, it depended on the intent to harm or help the listener. There are many cases of very positively evaluated lies (lying to the Gestapo) which are still nonetheless lies. There are also negatively evaluated truths (Fu et al., 2010; Fu et al., 2001). That said, there is some relationship between evaluation and categorization, as Fu et al. (2001) showed that Chinese adults who categorized modesty-motivated misrepresentations as lies also 
rated them more negatively. Studies that explicitly examine how evaluations of misrepresentations impinge on their categorization and vice versa would hopefully clarify how the two interact. Coleman and Kay's prototype would be particularly helpful here as it identifies "halfway" statements which are variously categorized as lies and non-lies in the same population - useful variation for examining the relationship of differential categorization to how misrepresentations are evaluated.

How do people mark, switch, and negotiate expectations about misrepresentation? In every cultural setting there is a default communicative context, as has been proposed for Grice's Cooperative Principle in the U.S. or some form of politeness principle in China. If this varies cross-culturally, do people universally come to interactions with a "truth bias"—an expectation that a partner will help one with the truth? Is there a culture where a Competitive Principleusually relegated to sports, games and politicsin the U.S.- - actually defines the default context which governs day-to-day interactions? Are there a limited number of these principles or rule sets cross-culturally? How do people mark or signal that the context, and thus expectations about misrepresentation, has changed? How do speakers strategically manipulate and revise these expectations, for example by reframing a lie as a joke, in different settings (Nachmann, 1984)? And what checks are in place to prevent such strategic shifts, such as the announcements in an airport stating that people making "inappropriate jokes" (presumably about bombs) will be detained?

Conclusion. People differ dramatically in how they think about lying and deception. These differences are interesting in their own right, but they also provide potentially important raw material for broader discussions, such as how people categorize acts into moral categories 
(lies vs. non-lies) and how different communicative principles (e.g., whether one should be informative, polite, or competitive) come into play in different contexts. But the rare focused case studies in places such as China and Southern Belize provide only tantalizing glimpses in the depth and breadth of human variation. Hopefully, careful descriptions in diverse contexts as well as new theories attempting to explain existing variation will fill out the full picture of diversity in how people around the world categorize and evaluation misrepresentations, lies, and deceptions.

Acknowledgments. I am grateful to Steven Neuberg, Jennifer Fewell, Joe Hackman, and Mariya Voytyuk for helpful comments on an earlier draft of the chapter. I also acknowledge support from the National Science Foundation grant BCS-1150813 and the University of Chicago and Templeton Foundation New Science of Virtues grant.

\section{References}

Arico, A. J., \& Fallis, D. (2013). Lies, damned lies, and statistics: and empirical investigation of the concept of lying. Philosophical Psychology, ahead-of-print, 1-27.

Ariely, D., \& Norton, M. I. (2007). Psychology and Experimental Economics: a gap in abstraction. Current Directions in Psychological Science, 16(6), 336-339.

Blum, S. D. (2007). Lies that Bind: Chinese Truths, Other Truths: Rowman and Littlefield.

Bok, S. (1978). Lying: Moral Choice in Public and Private Life. New York: Pantheon.

Carson, T. L. (2006). The definition of lying. Nous, 40, 284-306. 
Cole, S. A. N. (1996). Semantic prototypes and the pragmatics of Lie across cultures. The LACUS Forum, 23, 475-483.

Coleman, L., \& Kay, P. (1981). Prototype semantics: the English word lie. Language, 26-44.

Condon, J. C., \& Yousef, F. S. (1974). An Introduction to Intercultural Communication: Bobbs-Merril.

Danziger, E. (2010). On trying and lying: cultural configurations of Grice's Maxim of Quality. Intercultural Pragmatics, 7(2), 199-219.

Davis, D. D., \& Holt, C. A. (1993). Experimental Economics. Princeton, NJ: Princeton University Press.

Eichelberger, J. (2012). A Semantic and Pragmatic Analysis of the Spanish Word Lie: Implications and Applications for the Second Language Learner. Masters Thesis, Baylor University.

Friedl, E. (1962). Vassilika: A Village in Modern Greece. New York: Holt, Rinehard \& Winston.

Fu, G., Brunet, M. K., Lv, Y., Ding, X., Heyman, G. D., Cameron, C. A., \& Lee, K. (2010). Chinese Children's Moral Evaluation of Lies and Truths-Roles of Context and Parental Individualism-Collectivism Tendencies. Infant and Child Development, 19(5), 498-515.

Fu, G., Lee, K., Cameron, C. A., \& Xu, F. (2001). Chinese and Canadian adults' categorization and evaluation of lie- and truth-telling about prosocial and antisocial behaviors. Journal of Cross-cultural psychology, 32(6), 740-747. 
Fu, G., Xu, F., Cameron, C. A., Heyman, G. D., \& Lee, K. (2007). Cross-cultural differences in children's choices, categorizations, and evaluations of truths and lies. Developmental Psychology, 43(2), 278-293.

Gilsenan, M. (1976). Lying, honor and contradiction. In B. Kapferer (Ed.), Transaction and Meaning: Directions in the Anthropology of Exchange and Symbolic Behavior (pp. 191-219). Philadelphia: Institute of the Study of Human Issues.

Grice, H. P. (1989). Studies in the Way of Words. Cambridge, MA: Harvard University Press.

Handmann, M. E. (1983). La violence et al ruse: Hommes et femmes dans un village Grec. Aix-en-Provence: Edisud.

Hardin, K. J. (2010). The Spanish notion of Lie: Revisiting Coleman and Kay. Journal of Pragmatics, 42(12), 3199-3213.

Henrich, J., Heine, S. J., \& Norenzayan, A. (2010). The weirdest people in the world? Behavioral and Brain Sciences, 33(2-3), 61-83.

Hertwig, R., \& Ortmann, A. (2008a). Deception in experiments: revisiting the arguments in their defense. Ethics and Behavior, 18, 59-82.

Hertwig, R., \& Ortmann, A. (2008b). Deception in social psychological experiments: two misconceptions and a research agenda. Social Psychology Quarterly, $71(3), 222-227$.

Heyman, G. D., Sweet, \& Lee, K. (2009). Children's reasoning about lie-telling and truth-telling in politeness contexts. Social Development, 18(3), 728-746.

Hruschka, D. J. (2010). Friendship: Development, Ecology and Evolution of a Relationship. Berkeley, CA: University of California Press. 
Lakoff, R. T. (1973). The Logic of Politeness: Minding your p's and q's. In C. Corum, T. C. Smith-Stark \& A. Weiser (Eds.), Papers from the Ninth Regional Meeting of the Chicago Linguistic Society. (pp. 292-305). Chicago, IL: Chicago Linguistics Society.

Lee, K., Cameron, C. A., Xu, F., Fu, G., \& Board, J. (1997). Chinese and Canadian children's evaluations of lying and truth telling: similarities and differences in the context of pro-social and antisocial behaviors. Child Development, 68(5), 924-934.

Lee, K., \& Ross, H. J. (1997). The concept of lying in adolescents and young adults: Testing Sweetser's folkloristic model. Merrill-Palmer Quarterly, 255-270.

Lee, K., Xu, F., Fu, G., \& Cameron, C. A. (2001). Taiwan and Mainland Chinese and Canadian children's categorization and evaluation of lie- and truth-telling: A modesty effect. British Journal of Developmental Psychology, 19, 525-542.

Levine, T. R., Asada, K. J. K., \& Lindsey, L. L. M. (2003). The Relative Impact of Violation Type and Lie Severity

on Judgments of Message Deceitfulness. Communication Research Reports, 20(3), 208-218.

Ma, F., Xu, F., Heyman, G. D., \& Lee, K. (2011). Chinese children's evaluations of white lies: weighing the consequences for recipients. Journal of Experimental Child Psychology, 108(2), 308-321.

Mahon, J. E. (2008). Two definitions of lying. International Journal of Applied Philosophy, 22(2), 211-230. 
McCornack, S. A., Levine, T. R., Solowczuk, K. A., Torres, H. I., \& Campbell, D. M. (1992). When the alteration of information is viewed as deception: An empirical test of information manipulation theory. Communication Monographs, 59, 17-29.

Nachmann, S. R. (1984). Lies my informant told me. Journal of Anthropological Research, 40(4), 536-555.

Park, H. S., \& Ahn, J. Y. (2007). Cultural Differences in Judgment of Truthful and Deceptive Messages. Western Journal of Communication, 71(4), 294-315.

Seiter, J. S., Bruschke, J., \& Bai, C. (2002). The acceptability of deception as a function of perceivers' culture, deceiver's intention, and deceiver-deceived relationship. Western Journal of Communication, 66(2), 158-180.

Sweetser, E. E. (1987). The definition of lie: An examination of the folk models underlying a semantic prototype. In D. Holland \& N. Quinn (Eds.), Cultural Models in Language and Thought (pp. 43-66). Cambridge: Cambridge University Press.

Trompenaars, F. (1998). Riding the Waves of Culture. New York: McGraw Hill. Vrij, A. (2000). Detecting lies and deceit: the psychology of lying and the implications for professional practice: John Wiley \& Sons.

Wang, X., Bernas, R., \& Eberhard, P. (2012). When a Lie Is Not a Lie: Understanding ChineseWorking-Class Mothers' Moral Teaching and Moral Conducts. Social Development, 21(1), 68-87.

Xu, F., Luo, Y. C., Fu, G., \& Lee, K. (2009). Children's and adults' conceptualization and evaluation of lying and truth-telling. Infant and Child Development, 18(4), 307-322. 
Yeung, L. N. T., Levine, T. R., \& Nishiyama, K. (1999). Information Manipulation Theory and Perceptions of Deception in Hong Kong. Communication Reports, 12(1), 1-11. 